\title{
Norois
}

Environnement, aménagement, société

$191 \mid 2004 / 2$

Les types de temps

\section{COSTEL et les « Types de temps »}

COSTEL and the Weather types

\section{Vincent Dubreuil et Olivier Planchon}

\section{(2) OpenEdition}

Journals

Édition électronique

URL : http://journals.openedition.org/norois/1000

DOI : $10.4000 /$ norois. 1000

ISBN : 978-2-7535-1539-0

ISSN : $1760-8546$

Éditeur

Presses universitaires de Rennes

Édition imprimée

Date de publication : 1 mars 2004

Pagination : 11-13

ISBN : 978-2-86847-977-8

ISSN : 0029-182X

Référence électronique

Vincent Dubreuil et Olivier Planchon, « COSTEL et les « Types de temps » », Norois [En ligne],

191 | 2004/2, mis en ligne le 27 août 2008, consulté le 03 mai 2019. URL : http://

journals.openedition.org/norois/1000 ; DOI : 10.4000/norois.1000

Ce document a été généré automatiquement le 3 mai 2019.

(c) Tous droits réservés 


\title{
COSTEL et les « Types de temps »
}

\author{
COSTEL and the Weather types
}

\author{
Vincent Dubreuil et Olivier Planchon
}

1 En choisissant l'Université de Rennes 2 et le laboratoire COSTEL (Climat et Occupation du Sol par Télédétection) pour la tenue de ses journées 2003, la commission «Climat et Société » du Comité National Français de Géographie est venue conforter l'importance de ce site dans le "paysage climatologique » national. En outre, le fait d'aborder le thème des « types de temps " à Rennes et à COSTEL apparaît, avec le recul, comme la rencontre réussie entre une certaine tradition, de nouveaux développements méthodologiques et un renouvellement des questionnements scientifiques.

2 La longue tradition des recherches universitaires en climatologie à Rennes n'a pas d'équivalent dans la France de l'ouest. Les travaux initiés par C.P.PEGUY et poursuivis ensuite à l'ER-30 de Grenoble ont connu une résonance particulière dans notre région comme l'illustre la couverture (hélas incomplète) des Cartes Climatiques Détaillées de la France. On doit surtout à J.MOUNIER d'avoir ancré définitivement les recherches en climatologie à COSTEL dés le début des années 80 et d'avoir su les faire reconnaître, audelà de la communauté des géographes, au niveau international et dans le domaine des utilisateurs des images satellites: la meilleure illustration en a été l'organisation du $3^{\mathrm{e}}$ colloque de l'Association Internationale de Climatologie avec l'antenne ORSTOM du Centre de Météorologie Spatiale de Lannion en 1990. Plus récemment, les journées de la commission de climatologie de mai 1997, organisées à Rennes par V.DUBREUIL et J.P.MARCHAND, et la sortie aux PUR de l'ouvrage Le climat, l'eau et les hommes sont venues rappeler l'importance de ce thème dans les activités de recherche de l'équipe. A Rennes, l'étude des « types de temps » a d'ailleurs donné lieu très tôt à des publications majeures, un panorama complet des connaissances et du lien entre «types de temps » et « types de circulation " étant dressé dans la thèse de J. MOUNIER ${ }^{1}$. Par la suite, la thèse de J.P.MARCHAND replace leur utilisation dans le cadre d'une approche conceptuelle plus résolument tournée vers l'analyse spatiale et sociale.

3 C'est à partir de ce solide corpus théorique et méthodologique et d'une approche jamais éloignée des questions de société que se sont développés les travaux de l'équipe COSTEL. 
Les thèses soutenues depuis 1980 montrent que si «les types de temps " ne sont que rarement au cœur de la problématique de recherche, leur étude n'est jamais occultée mais plutôt repositionnée dans une démarche plus tournée vers leurs conséquences (et notamment les risques liés aux sécheresses, inondations, tempêtes). Surtout, l'apport des bilans de l'eau et de l'imagerie satellitaire (notamment dans le cadre du Programme National de Télédétection Spatiale) permet de passer d'une approche analytique et subjective à une quantification plus rationnelle des processus comme l'illustrent les titres des thèses en rapport avec le climat soutenues à COSTEL depuis une quinzaine d'années :

BERHAULT Yvon (1989). - Contraintes et potentialités de l'écoulement fluvial en Bretagne. Essai de modèlisation des basses eaux.

ROBERT-LUCIANI Thierry (1990). - Les pluies dans le Frioul, les situations à l'origine de leur abondance. Une approche par l'image satellitaire.

PERRON Gilles (1990). - Types de temps et ensoleillement en climat océanique. Une méthode de climatologie appliquée à la Bretagne.

ATTILLAH Abderrahman (1993). - Les influences maritimes sur le climat du Maroc atlantique central. Une analyse par télédétection spatiale.

DUBREUIL Vincent (1994). - La sécheresse dans la France de l'Ouest: Etude d'après les bilans hydriques et les données des satellites NOAA.AVHRR.

DEMRI Zahra (1995). - Variabilité interannuelle de la sécheresse et de l'humidité au Maroc.

LE MASSON Loic. - (1999) Vents et tempêtes sur le littoral de l'Ouest de la France: variabilité, variation et conséquences morphologiques.

BONFANTI Godlive (2003). - Les bâtiments face aux cyclones tropicaux: analyses comparées d'un indicateur de la vulnérabilité en région Caraibe.

Depuis quelques années, un certain renouvellement des questionnements scientifiques peut aussi être souligné concernant l'étude du climat (à COSTEL et ailleurs) :

1. le climat n'est plus considéré comme stable (ce qui peut relativiser un certain nombre de résultats antérieurs basés sur la stationnarité du climat...). Plusieurs travaux de l'équipe ont ainsi montré comment s'inscrivait la France de l'ouest dans le cadre du "global change »: augmentation des températures, changement de direction des vitesses de vent maximales enregistrées lors des tempêtes, la tendance la plus spectaculaire étant l'accentuation du déficit estival centré désormais sur le mois d'août.

2. Ces évolutions constatées ne sont-elles pas aussi, en partie, une conséquence des actions humaines à l'échelle globale ou régionale? Quel est le rôle des modifications apportées aux paysages ruraux et par les nouvelles pratiques culturales? C'est ici l'importance des phénomènes d'interaction entre la surface de la terre (ou de la mer) et l'atmosphère qui est soulignée: on sait notamment comment le développement des agglomérations modifie le climat. Mais à l'échelle régionale, les interactions continent-océan génèrent également des circulations et des types de temps spécifiques (brise de mer) et sur d'autres espaces, où les changements d'occupation du sol sont encore plus spectaculaires, comme en Amazonie, c'est toute la question de l'impact climatique de la déforestation qui est posé.

3. L'élargissement thématique et institutionnel de la recherche oblige aussi les géographes climatologues à mieux formuler leur questionnement scientifique. Dans le cadre d'une UMR multisite du CNRS comme celle à laquelle appartient désormais COSTEL, c'est la question de l'eau et des «bassins versants» qui est privilégiée, ce qui suppose une approche plus «finalisée » du concept de «type de temps». La collaboration avec des biologistes ou des écologues dans le cadre de l'Institut Fédératif CAREN (Centre Armoricain de Recherche en Environnement) pose la question du climat vécu ou perçu non seulement pas les êtres humains mais aussi par les plantes et les animaux. La collaboration avec des physiciens pour la modélisation numérique du climat ou avec des agronomes (en France ou en Afrique du 
Sud) conduit aussi à poser la question du type de temps et de ses conséquences en des termes plus concrets.

5 Ce développement des recherches, appuyées ou non sur des structures institutionnelles, ne doit pas faire oublier au géographe climatologue son objet de recherche: il l'amène cependant à mieux préciser ses concepts pour pouvoir dialoguer avec d'autres disciplines.

\section{NOTES}

1. Voir également: J.MOUNIER, 1977: Le type de temps, un choix pour le géographe: climatologie synoptique ou climatologie compréhensive; Actes des journées Nationales de climatologie ; Cahier $n^{\circ} 7 \mathrm{du}$ Centre de Recherche de Climatologie de Dijon, pp.99-117

\section{RÉSUMÉS}

Les recherches en climatologie au laboratoire COSTEL sont une tradition ancienne: les premiers travaux ont été menés sous l'impulsion de C.P. Péguy et J.Mounier, ce dernier ayant contribué au rayonnement international de l'équipe et à son inscription dans les programmes de télédétection. Sous la direction de J.P.Marchand, COSTEL a centré ses études sur les types de temps « à risque » dans le cadre d'une approche spatiale proche des préoccupations sociales. Les recherches actuelles privilégient les collaborations pluridisciplinaires et une ouverture internationale encore plus affirmée et diversifiée.

Climate research at the COSTEL laboratory started with the works carried by Pr. C.P. Péguy in the 50's and Pr. J. Mounier. J. Mounier contributed to the international influence of the COSTEL team and to its participation in remote sensing research programmes. Later on, with Pr. J.P. Marchand at the head of the COSTEL laboratory, researches on weather types developed with a spatial approach of the risks as well as a high interest for the social concerns. The present researches take multidisciplinary co-operation and stronger and more diversified international relations into account.

\section{AUTEURS}

VINCENT DUBREUIL

COSTEL, UMR 6554 CNRS (LETG), Université Rennes 2, Vincent.dubreuil@uhb.fr

\section{OLIVIER PLANCHON}

COSTEL, UMR 6554 CNRS (LETG), Université Rennes 2, olivier.planchon@uhb.fr 